\title{
HP HEROES
}

\section{CANADIAN SOCIETY OF INTERNAL MEDICINE}

By Bert Govig, MD

\section{About the Author:}

Dr. Bert Govig is an Assistant Professor of Medicine at McGill University and Chief of Internal Medicine at the Centre Hospitalier Hotel Dieu d'Amos. He has a consult-based practice and is skilled in the techniques of bronchoscopy, echocardiography, and cardiac pacing. He is director of the Cardiac Rehabilitation Program at the Centre Hospitalier Hotel Dieu d'Amos.

Submitted: April 4, 2019. Accepted: April 24, 2019. Published: May 21, 2019. DOI: 10.22374/cjgim.v14i2.374

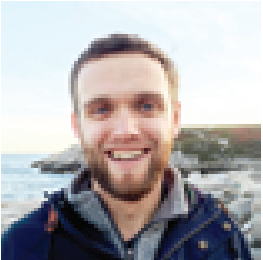

Hi Tommy - winning the Hui Lee Scholarship as an R-3 is quite impressive. Tell us what path you took you to bring you to such auspicious heights so early in your career.

I'm an internal medicine resident at Dalhousie University, based in Halifax. I'm $13^{\text {th }}$ generation Nova Scotian, but also grew up in North Carolina, Ottawa, and New Hampshire before moving back to Halifax.

I come from a medical family which has hugely influenced me. My mom is a geriatric psychiatrist who did rural outreach for much of her career. My dad was a pioneering interventional neuroradiologist, and my uncle was an AIDS physician in the early days of the crisis.

Inside medicine, I'm interested in the health of people who are socially excluded and marginalized, including people who use substances and people experiencing homelessness. I've been getting some extra training in addiction medicine as I go along, and I try to incorporate that within my internal medicine practice. Approaches like motivational interviewing and harm reduction seem to be just as useful when caring for my patients with diabetes and COPD as they are for my patients who are street-involved with HIV and opioid use disorder.

Outside medicine, I love playing music with friends, telling stories (especially about when I used to play rugby), and trying to stay active despite many lingering musculoskeletal injuries.

\section{What sparked your interest in Health Promotion?}

I went into medicine in part because of my interest in social justice. Early on in medical school I was fortunate to meet
Patti Melanson, a nurse and founder of Mobile Outreach Street Health (MOSH), Halifax's street nursing service. Following her and her team in shelters, rooming houses, and on outreach with Mainline needle exchange, I got to meet people with multiple complex medical conditions, often complicated by psychiatric, cognitive, and addiction issues, who had amazing survival skills but could not participate in our traditional health care system. MOSH's approach is based around relationships with clients, meeting them where they are, learning what they need, and trying to help in any positive way they can with compassion and non-judgment. They acknowledge the broader structural influences on peoples' health, including poverty, stigma, racism, housing, and drug criminalization. I saw people improve control over their health when offered supports like housing, food, sterile drug consumption equipment, and peer outreach along with access to health care.

This often felt like quite a different approach than what I was learning in the classroom and on the wards in our tertiary care, academic centres. In clerkship I took care of some of these same patients when they were hospitalized with serious illnesses. It felt like we had little to offer and often made things worse by forcing people into opioid withdrawal and discharging them against medical advice. The difference in approach kind of blew my mind. I was fortunate to have other amazing mentors in medicine and research throughout medical school to help me make sense of these experiences, including Drs. Ken Rockwood, Colin Van Zoost and Susan Kirkland, who helped me develop some projects to make things better. I also did electives related 
to health care of the homeless and addiction medicine in Ottawa, Toronto, and Vancouver to experience different approaches.

\section{Describe your current HP-related work}

These experiences motivated me to try to see how our health care system could reach out and support people who are socially excluded, including incorporating the approaches and expertise of our community-based organizations into the formal health care system and bringing the resources of our tertiary care, academic centres into the community.

Our current clinical and research projects involve bringing addiction medicine and harm reduction into the hospital, and doing internal medicine specialist consultation outreach. After getting training as an opioid agonist therapy prescriber, I've started seeing inpatients with opioid use disorder and reviewing these cases with our community-based addiction medicine physicians. This has not only been getting people onto treatment for opioid use disorder, but helping people stay in hospital to complete the treatment of the serious medical problem that led to their admission. We've changed hospital policies to include takehome naloxone kit distribution, and developed a protocol for emergency department-based initiation of buprenorphine. I'm working with a community group to develop a proposal for a safe injection site in Halifax, and bring resources from our tertiary care centre including testing and treatment for HIV and hepatitis C. We're also building an internal medicine consultation service with $\mathrm{MOSH}$, to assess and help manage their most medically complex clients - many of whom are aging and prematurely facing dementia, incontinence, and mobility impairment. We've been doing education sessions with residents, students, nurses, pharmacists, and social workers on harm reduction, addictions, and homelessness in acute care.

\section{Name three things outside of medicine that make your heart go pitter patter (sports, hobbies, passions, odd interests).}

Banjo music, game-winning rugby drop goals, and well-attended kitchen parties

\section{If you had a budget to send books to the entire CSIM membership, what would books would you choose and why? (up to 3)}

- Medicine Unbundled: A Journey through the Minefields of Indigenous Health Care by Gary Geddes. This history of Indigenous health care in Canada is painful to read but is beautifully and powerfully written. Mr. Geddes explores past and present health and health care inequities in Canada, including the history of our "Indian Hospitals" - a segregated health care system parallel to the system of residential schools.
- Fighting for Space: How a Group of Drug Users Transformed One City's Struggle with Addiction by Travis Lupick. This is the captivating story of the Portland Hotel Society, the Vancouver Area Network of Drug Using People (VANDU) and the history of harm reduction and drug user activism in Vancouver's Downtown East Side. The organization and advocacy of people who use drugs for health services, policy and law changes, and to save lives is a huge inspiration for me.

- An Unquiet Mind by Kay Redfield Jamison. Dr. Redfield Jamieson is a psychologist and bipolar disorder researcher, who has bipolar disorder herself. In her memoir, she has a beautiful way of describing why its human nature to make choices that are not in our own long-term best interest, for example skipping some lithium and precipitating a manic episode in order to make a grant deadline.

\section{What "secret" (or relatively unknown) Canadian vacation would you recommend to readers?}

An August day in Nova Scotia's Annapolis Valley - touring the wineries, walking the Bay of Fundy beaches, tubing down the Gaspereau River, and getting lost in Wolfville's book stores and coffee shops.

\section{What traits do you most appreciate in your colleagues?}

Kindness, patience, and enthusiasm.

\section{What clinical moments or acts make you feel most in the groove of your professional vocation?}

- Reasoning out a complex diagnosis

- Starting a hospital inpatient on opioid agonist therapy

- Working with a patient to figure out a practical treatment plan incorporates the reality of their life, resources, and priorities

\section{If you had a magic wand and could impose a single condition or behaviour on all of the population to improve overall health - what would it be?}

Adequate income - so that people don't have to live in "survival mode" all the time, and can have the peace and freedom to make real choices

Thank you Tommy for sharing and inspiring us all. If you were touched or inspired by Dr. Brothers' journey, if you read one of his recommended books, or want to thank him for his contributions, reach out to him in person at the CSIM meeting in Halifax. 